\title{
Consideraciones previas a una teoría de la verdad"
}

\author{
Óscar Augusto García Zárate \\ Universidad Nacional Mayor de San Mercos
}

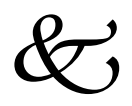

\begin{abstract}
Restmen
Sobre la base de considerar que la verdad es una propiedad, el autor sostiene que se predica de proposiciones, creencias, afirmaciones y oraciones, en el orden jerárquico correspondiente. Asimismo, presenta un bosquejo de tres teorías de la proposición, y concluye que es indiferente declararse a favor de cualquiera, ya que todas consideran a las proposiciones como entes privilegiados con respecto a la atribución del predicado 'verdadero'. Finalmente, arguye que un problema tan complejo como la elaboración de una teoría de la verdad es el de una teoría de las proposiciones.

Palabras claves: Verdad, propiedades, proposición, filosofía del lenguaje, filosofíade la lóoica.
\end{abstract}

\section{Introducción}

T a verdad no es una sustancia y, con el objeto de no incurrir en Leificaciones, quien la estudie como problema debe estudiar tan sólo lo que sea verdadero. Un análisis tanto del lenguaje natural como del formal indica lo anterior, pues la verdad se emplea como predicado, lo que denota una propiedad. La verdad es, por tanto, una propiedad.

Ahora bien, ¿qué es una propiedad? En la siguiente sección se ofrece una respuesta a esta pregunta. También se presenta una clasificación de los diversos tipos de propiedades; sin embargo, no se señala qué clase de propiedad es la verdad, tal cosa se realiza en la segunda sección, en donde se sugiere que es afín a las relaciones. 


\section{Propiedades}

Se dice que las propiedades son atributos, cualidades o características de las cosas. Así, el color es una propiedad, el amari 7 lo es otra, mientras que ser colory ser amarillo son dos más². Aparte de esto, se sostiene que las propiedades son o universales o particulares. En el primer caso, las propiedades, a diferencia de los particulares, son entes que pueden ser ejemplificados infinitas veces, cada una de ellas por entes particulares diferentes; mientras que en el segundo, las propiedades son individuos, tanto como los individuos de los cuales son propiedades. De esta manera, el amarillo de dos globos es distinto en número, pues se da la amarillez del primer globo y la amarillez del segundo globo, y así de cuantas cosas se digan amarillas. Quienes identifican las propiedades con universales se conocen como realistas; quienes lo hacen con particulares, como nominalistas. Aquellos abogan por la existencia dojetiva de las propiedades; estos, por la tesis contraria.

La versión dominante del nominalismo contemporáneo es la quineana [ (Goodman y Quine 1947) (Quine 1960: 90 y s. .) ] . Según ésta, un predicado se refiere al conjunto total de dojetos para los cuales es verdadero. Así, si yo digo:

\section{(1) Mi globo es amarillo,}

habré dicho una oración verdadera si y sólo si mi globo es miembro del conjunto de cosas amarillas, por lo cual el predicado, al tener referencia múltiple, aparece como término general, en oposición al nombre que es término singular, pues se refiere a un solo objeto. El realismo señalaría que los predicados también se refieren a un solo objeto, esto es, a la propiedad en cuestión, propiedad que no es un conjunto de dojetos, sino, antes bien, el atributo que forma un conjunto. Para esta postura, tanto el nombre como el predicado son términos singulares, y (1) es una oración verdadera si y sólo si mi globo tiene la propiedad a la que hace referencia el predicado, esto es, la amarillez. En una palabra, los nombres denotan objetos y los predicados denotan propiedades ${ }^{3}$.

Hechas las distinciones terminológicas del caso, es preciso señalar las diferentes clases de propiedades ${ }^{4}$. Por supuesto, no se presupone 
la postura quineana, de lo contrario, se incurriría en un regreso ad infinitum, pues, al entenderse a las propiedades como clases, luego de haberse asado hablar de clases de propiedades, se solicitaría las clases de clases de propiedades, luego, las clases de clases de clases de propiedades, etc.

\subsection{Propiedades según su jerarquía}

Es posible establecer una clasificación tomando en cuenta la estructura lógica de las propiedades. De esta manera, podemos hablar de propiedades de primer orden, propiedades de segundo orden, propiedades de tercer orden, etc. Se parte por establecer que aquellas propiedades que sólo pueden ser ejemplificadas por individuos pertenecen al primer orden de nuestra jerarquía. Iuego, se debe considerar que estas propiedades pueden tener propiedades, es decir, ser ejempl ificaciones de otras propiedades, a las aules se 1 lamará de segundo orden. Por ejemplo, la amarillez es una propiedad de primer orden que ejemplifica la propiedad de ser un color, la cual es una propiedad de segundo orden. De continuarse esta cadena, se establecería que la propiedad de ser un color es una ejemplificación de la propiedad de ser una sensación 5 , la cual sería una propiedad de tercer orden.

Es cierto que una propiedad puede ejemplificarse a sí misma; así, la propiedad de ser una propiedad es ella misma una propiedad, pero, si no se toman las precauciones necesarias, podría aparecer la conocida paradoja de Russell. Por ejemplo, la expresión predicativa 'es una propiedad que no se ejemplifica a sí misma' denota una propiedad. Si ésta se ejemplifica a sí misma, entonces es una propiedad que no se ejemplifica a sí misma, y si no se ejemplifica a sí misma, entonces se ejemplifica a sí misma. Una inconsistencia semejante sólopuede ser resuelta por la teoría de las tipos propuesta por Russell a propósito de esto.

\subsection{Relaciones}

Las relaciones son propiedades que se predican de por lo menos dos individuos. De esta manera, la relación de ser más rápido que es 
una relación que involucra dos individuos, como también lo es la relación de conversar. Estas relaciones son conocidas como binarias. Por otro ladb, cuando una relación involucra tres individuos se le conoce como temaria. Ejemplo de una relación temaria es la de estar entre. Así,

(2) Perú está entre Ecuador y chile.

La lista de relaciones que involucren más individuos es infinita, aunque enumerable, pues es idéntica a la del conjunto de números positivos.

\section{3. Propiedades de grado múltiple}

Se dice que una propiedad es de grado múltiple cuando puede ser verdadera de varios particulares, numéricamente distintos, y variables en número. Por ejemplo, la propiedad que denota la expresión predicativa 'fuercon presidentes del Perú' es verdadera de Alberto Frjimori y Ramón Castilla, así como de Nicolás de Piérola, Femando Belaúnde y Manuel Odría, también de Valentín Paniagua, José de la Riva Agüero, Óscar Benavides, Alan García y Manuel Prado.

\section{4. Propiedades particularizadoras}

Las propiedades particularizadoras son análogas a los números, pues permiten contar objetos. Así, las propiedades de ser una jaula y ser un león son propiedades particularizadoras, ya que permiten que se especifique cuántas jaulas hay en el zoológico, y cuántos leones hay dentro de cada una de ellas. Las propiedades particularizadoras se refieren, por tanto, a nombres commes.

\subsection{Propiedades caracterizadoras}

A diferencia de las propiedades particularizadoras, las propiedades caracterizadoras no dividen el mundo en un número definido de cosas que pueda inventariarse. Las propiedades caracterizadoras como la amarillez no permiten contar las cosas del mundo, lo que contamos 
son cosas amarillas como globos o camisas; por ello, este tipo de propiedades se refiere a adjetivas.

\subsection{Género y especie}

Ia tradición filosófica occidental ha empleado los términos 'género' y 'especie' desde su época prístina. Ambos, género y especie, son propiedades relativas. El género es una propiedad general, y la especie se halla incluida dentro de él. Por ejemplo, ser mamúfero es una especie relativa al género ser animal, y un género relativo a la especie ser humano.

\subsection{Determinables y determinantes}

Una distinción similar a la de género y especie es aquella entre determinables y determinantes. El color y la forma son propiedades determinables, mientras que la amarillez y la triangularidad son propiedades determinantes. Al igual que la diferencia entre género y especie, la distinción entre determinables y determinantes es relativa: la amarillez es un determinante con respecto del color, y un determinable con respecto a la sombra específica de amarillo.

\subsection{Clases naturales}

Las clases naturales son propiedades que pretenden dar cuenta de divisiones reales de la naturaleza. Ejemplos típicos de estas propiedades son la de ser una clase específica de animal (perro, gato, leán, caballo, etc.) , y la de ser una clase específica de elemento químico (plata, aluminio, calcio, etc.) . Las clases naturales se oponen a las clases artificiales (ser una computadora, ser un lapicero). Estas propiedades sugieren mucho debate filosófico (Quine 1969) .

\subsection{Propiedades purramente cualitativas}

Si una propiedad depende de la existencia de un individuo, no es una propiedad puramente cualitativa. Por ejemplo, vivir en Lima y ser 
alcalde de Lima son propiedades que involucran la existencia de Lima, ente que pudo no haber existido y, en consecuencia, aquéllas tampoco. En este caso, las propiedades puramente cualitativas son vivir en y ser alcalde de, éstas no involucran la existencia de un ente definido.

\subsection{Propiedades esenciales y accidentales}

Una propiedad es esencial si un individuo la tiene en toda circunstancia posible de su existencia; si no sucede esto, es accidental o contingente. Mi gldbo es amarillo, pero pudo haber sido rojo; por ello, la amarillez es una propiedad accidental. La extensión, sin embargo, es una propiedad esencial de mi globo, pues siempre será un cuerpo extenso. También puede decirse que si algo es miembro de una clase natural, ser miembro de aquella clase es una propiedad esencial para aquello. Por ejemplo, León, mi gato, tiene la propiedad esencial de ser miembro de la clase natural de los gatos.

\subsection{Propiedades extrinsecas e intrínsecas}

Algunas propiedades se ejemplifican sólo cuando el indivicuo del cual se predica está en relación con otro. A estas propiedades se les llama extrínsecas. Por decirlo de algún modo, yo ejemplifico la propiedad de estar casado sólo porque firmé cierto contrato con mi mujer. En oposición a estas propiedades, aparecen las intrínsecas, las cuales se ejemplifican por individuos sin que sea necesario que estos se relacionen con otros. Un ejemplo es la propiedad de pesar $85 \mathrm{ki}$ logramos. Es cierto, sin embargo, que luego de un análisis se puede identificar que muchas propiedades -si no todas- son relacionales.

\section{12. Propiedades primarias y secundarias}

La influencia lockeana admite la distinción entre propiedades primarias y secundarias (Locke 1987: 11, viii, 15) . De acuerdo con esto, las propiedades primarias son características dojetivas del mundo. 
Entre ellas están la forma, el tamaño y la masa. Las propiedades secundarias, por su lado, son características que dependen del sujeto cognoscente; entre éstas se incluyen el color, los sabores, los sonidos y los olores.

\section{Entes verdaderos}

Un conjunto puede ser cal ificado de infinito. Así también, hay casas que pueden ser calificadas de verdaderas. Ia infinitud se manifiesta a través de un conjunto infinito, y la verdad a través de algo que sea verdadero. Infinitud y verdad son, pues, propiedades, Y, en cuanto tales, sólo pueden manifestarse a manera de predicados atribuidos a ciertos sujetos. El problema de quien teorice acerca de la verdad y la infinitud empieza con el establecimiento del conjunto de entes a los cuales quepa predicárseles con legitimidad 6 . Luego de haber llevado esto a término, se conocerá qué puede ser verdadero o infinito, y qué no.

El propósito de esta sección es definir el conjunto de entidades que pueden ser verdaderas o no verdaderas, es decir, falsas. Como complemento, podrá inferirse que todo aquello que no figure en la enumeración aducida, no podrá ser verdadero ni falso7.

En principio, tras haberse dicho que la verdad (o falsedad) es una propiedad, y que lo buscado son los sujetos que la muestren, se deberá afimar que entre estos sujetos no figuran los entes físicos. Esto parece ser una trivialidad; sin embargo, no lo es del toob, pues quien sastenga que la puerta es verdadera, o, acaso, que los duendes no lo son, no será tomado como alguien que emite incoherencias. Tanto la puerta como los duendes son sujetos que se presumen referidos a un ente físico, y, por lo común, de estos se predica la falsedad, mientras que de aquélla la verdad. En ambos casos, el sentido de los hablantes resulta ser manifiesto para su comunidad lingüŕstica, aunque no coincida con el que es propio de la verdad. Quien sostenga que la puerta es verdadera, sostiene que la puerta está allí, esto es, que no es una ilusión, que es algo existente. Quien, por otro lado, sostenga que los duendes no son verdaderos, sostiene que los duendes no existen. Así, 
la verdad se emplea como concepto intercambiable en relación con el de existencia, y éste, a su vez, con el de naturaleza real. No dostante su carácter de primitivos, es posible trazar múltiples distinciones entre estos tres conceptos, las mismas que no tienen lugar en este artículo, pues, para sus propósitos, basta señalar que aquello que existe son entes cuya existencia no se establece en virtud de otros entes de estructura formal disímil, cosa que sí sucede en el caso de lo que es verdadero8. Por lo demás, el hecho de que la existencia pueda considerarse como predicado aparte de propiedad es algo cuestionable desde la crítica kantiana del argumento ontológico (Kant 1998: A592602 ( B620-630), mientras que la verdad es una propiedad al mismo tiempo que un predicado lógica y gramaticalmente lícito?. La verdad, por tanto, no es cualidad de un ente físico, y es seguro que ella tampoco sea un ente o sustancia de estas características.

No originará mayor remuencia conceder que tan sólo cabe predicar la verdad de ciertas entidades lingüísticas, o, específicamente, de lo expresado por éstas, y también de estados mentales como, por ejemplo, creencias, dudas y deseos. La lista es bastante luenga, abarcaría desde las afimaciones, oraciones, enunciados y expresiones hasta las proposiciones, entidades abstractas que suelen identificarse con el significado, y que una línea de pensamiento que se remonta a Aristóteles (1967: 16a 9-18) encuentra como las privilegiadas para la atribución de verdad y falsedad. Este artículo habrá logrado la consecución de su cometido si señala las diferencias que se dan entre las entidades mencionadas de modo que pueda suscribir la tesis que sostiene que la proposición posee un derecho primario sobre la verdad, y que el de las otras no es sino consecuencia de éste.

Es conveniente empezar trazando una distinción entre el enunciar y lo enunciado, entre el afirmar y lo afirmado. Enunciados y afirmaciones son producto de enunciar y afirmar, respectivamente. Muchas enunciaciones pueden referirse al mismo enunciado; muchas afirmaciones, a la misma afirmación. Resulta obvio, entonces, que enunciados y afirmaciones no son actos espacio-temporales y únicos, sino el resultado de estos. Ahora bien, enunciados y afirmaciones pueden hacer las veces de creencias, asunciones, dudas, deseos y conjeturas. $Y$, al igual que enunciados verdaderos y afimaciones 
verdaderas, es correcto predicar la verdad de creencias, asunciones, dudas, deseos y conjeturas. Se observa, sin embargo, que aquello que prima son los enunciados, pues la afirmación lo es de un enunciado. Pero, ¿qué es un enunciado?

El concepto de enunciado se identifica de manera estrecha con el de proposición. Uno y otro pueden tomarse como el mismo, sólo si previamente se ha hecho notar que el enunciado no es una oración, evitándose el equívoco terminológico infeliz en el que no pocos traductores incurren. Un enunciado es una entidad abstracta, y una oración, un signo, una entidad física. La oración, en tanto conjunto de palabras inscritas sobre una superficie o en tanto sonido, se diferencia del acto de emitirla, escribir o hablar; éste es un proceso, aquella una entidad; ambos, no dostante, son constituciones físicas. En este sentido, se concede que dos sujetos, A y B, emitan la misma oración. El sujeto Adice:

(a) Lima es la capital de Perú;

el sujeto Bdice:

(b) Lima es la capital de Perú.

Ahora bien, (a) y (b) son entidades físicas únicas e irrepetibles. Estas entidades físicas, sin embargo, tienen una configuración idéntica, son dos expresiones diferentes y numéricamente distintas, pero son la misma oración, como las mil copias de este artículo son individuos diferentes y numéricamente distintos, aun cuando sean el mismo artículo. Un tercer sujeto, $C$, pooría añadir:

(c) Lima ist die Hauptstadt von Peru.

(a) y (b) son expresiones diferentes de la misma oración; (c) es diferente de (a) como (b) es diferente de (a), y es diferente de (b) como (a) es diferente de (b) , pero no expresa la misma oración que (a) y (b) , aunque sí la misma proposición o enunciado. Al igual que la misma oración puede ser hecha por diferentes expresiones, oraciones diferentes pueden expresar (afirmar) la misma propasición. Por ejemplo, si durante el 25 de diciembre de 2005 el sujeto D dice:

(d) Hoy es Navidad; 
y durante el 31 diciembre de 2005 el sujeto E dice:

(e) Hace seis días fue Navidad.

Entonces, los sujetos Dy E afirman la misma proposición, y (d) y (e) expresan la mi.sma proposición. Pero si el sujeto $F$ dice sin que sea relevante cuándo:

(f) El 25 de diciembre de 2005 D dijo que era Navidad, el sujeto $F$ no afirma la misma proposición que $D$, esto es, (f) no expresa lo expresado por (d) , sino el acto por el cual fue expresado (d) . Por otro lado, advertido que muchas oraciones pueden expresar la misma proposición, es también cierto que la misma oración puede expresar diferentes proposiciones. Así, durante el 26 de diciembre de 2005 el sujeto Gdice:

(g) Hoy es Navidad.

El sujeto $G$ ha expresado la misma oración que el sujeto $D$, pero (g) no expresa la misma proposición que (d) .

En este punto, resulta claro cuál es la diferencia entre proposición, oración, expresión, afimación, creencia y demás. Las expresiones expresan oraciones; las oraciones, a su vez, expresan afirmaciones, creencias y otros estados mentales. Las proposiciones, en último término, son los entes que las afirmaciones o creencias expresan, es decir, aquello que se afirma o aquello que se cree. De este modo, si una proposición $P$ es verdadera, la creencia $R$, o la afimación $I$, que la expresa será verdadera; $\mathrm{Y}$, por consiguiente, la oración $O$ que exprese la afirmación $I$ o la creencia $R$ será verdadera ${ }^{10}$. No se puede, sin embargo, continuar esta cadena e inferir que las expresiones que correspondan a oraciones verdaderas, sean también verdaderas. La función de una expresión es servir de materia para una oración, la expresión es aquello que da naturaleza física a la oración, o, si se prefiere, aquello que la pone en acto. La verdad de una oración no puede, por tanto, adjudicarse a su expresión; de lo contrario, se cometería la falacia de análisis, ya que la verdad es propiedad de una oración, pero no de sus partes constituyentes, ni de las palabras ni de aquello que sea materia de éstas, y no hay ninguna razón para que lo sea ${ }^{11}$. El resultado de estas disquisiciones puede resumirse como sigue: 
1. Una oración O es verdadera si y sólo si la afimación I o la creencia $R$ expresadas por ella, expresan, a su vez, una proposición $P$ que sea verdadera.

2. Una oración O es falsa si y sólo si la afimación I o la creencia $R$ expresadas por ella, expresan, a su vez, una propasición P que sea false.

Es frecuente hablar de proposiciones en términos de la información semántica que las expresiones y oraciones tomadas en contextos particulares expresan (Cartwright 1962: 81-103). Pero, a pesar de esto, la proposición no es el significado de una oración como erróneamente se podría conjeturar a partir de lo anterior, sino considérense las expresiones de oraciones con significados diferentes que expresan la misma proposición (expresiones (d) y (e) ) , y las expresiones de oraciones con el mismo significado que expresan proposiciones diferentes (expresiones (d) y $(\mathrm{g})$ ) $^{12}$. Aunque estos contraejemplos sean definitivos, el problema de la identidad entre significado y proposición podría quedar abierto, pues no es de interés para los fines de este artículo, y si se le menciona de pasada, es porque el concepto de proposición está involucrado aunque de manera indirecta.

Las proposiciones se han revelado, pues, como aquello que se afirma y aquello que se cree, esto es, como aquello en donde se encuentra primariamente la verdad. En un segundo nivel están las afimaciones, creencias, dudas, deseos y demás estados mentales. Finalmente, las oraciones constituyen el tercer nivel de verdad, el cual, al igual que el segundo, depende de la verdad de las proposiciones que exprese. La interrogante que naturalmente se origina es de qué depende la verdad de una proposición, qué hace a una proposición verdadera. Desde una postura neutral, es imposible indicar si más allá de la proposición se encuentra otro objeto de expresión de cuyo hipotético valor de verdad dependa el de aquélla, pues hacerlo es tarea de una teoría de la verdad.

\section{La esencia de la proposición}

En la sección anterior se ha establecido la extensión del conjunto de entes de los cuales se predica la verdad con validez. Tras una serie 
de ejemplos y argumentos, conclú́ que las proposiciones poseen un derecho primario sobre la verdad, mientras que las actitudes proposicionales y las oraciones poseen uno derivado con rspecto al de aquéllas. Por supuesto, no me adjudico la patemidad de esta tesis, pues, aunque con trazas diferentes, se encuentra ya en la filosofía antigua, medieval y modema. Mi dojeto tan sólo fue aclarar las nociones en cuestión como paso previo al desarrollo de una teoría de la verdad propia. En este sentido, partí asumiendo que la verdad es una propiedad, premisa de la cual se desprendieron naturalmente las conclusiones que presenté. Ahora bien, a pesar de que esto resulte definitivo con respecto al problema que me propuse abordar, mis colaboradores se han permitido esgrimir una objeción que suponen devastadora al respecto. Ésta se reduce a que no defino de manera explícita el concepto de proposición que manejo, pues haber declarado que es aquello que es verdadero o falso, y que es objeto de creencia y afimación, sólo fue dar una característica, la cual -si bien es cierto definitoria- no entraña la esencia de una entidad tan particular y problemática como la proposición. En resumen, no habría ofrecido una respuesta a la pregunta obvia '¿qué es una proposición?' , Y, así, concluir que las proposiciones son los entes con privilegio sobre la verdad no sería algo fundado. Expresada de esta manera, afirmo que es una crítica que resulta irrelevante para mis propósitos, pues cualquiera que sea la respuesta que ofrezca, ésta siempre considerará a la proposición como aquello que tiene privilegio sobre la verdad, y hasta aquí mi fin no ha sido otro que decir cuáles son los entes verdaderos, y no señalar qué son los entes verdaderos. Admito, sin embargo, que no dar respuesta a un reparo tan previsible como éste, constituiría una omisión que posiblemente degenere en serios conflictos para mi teoría; por ello, considero necesario efectuar una réplica concluyente, de modo que pueda evitar cualquier tipo de suspicacia en el futuro.

Mi argumento consiste en presentar las diferentes respuestas que puedan aducirse a la pregunta por la esencia de la proposición, para, una vez llegado al final, hacer manifiesto que, a pesar de su mutua oposición, todas consideran a las proposiciones como sujetos privilegiados con respecto de la verdad. Mencionaré tres posiciones: 
la de los mundos posibles, la de las proposiciones estructuradas y la llamada explicación algebraica ${ }^{13}$. La primera redunda en implicancias con respecto a la relación entre proposición y oración, mientras que las das últimas no; por ello, me limito a dar cuenta simple de aquélla.

Los teóricos de los mundos posibles aseguran que las proposiciones son funciones que van desde los mundos posibles a los valores de verdad [ (Lewis 1973), (Stalnaker ¿?: 79-91) ], es decir, algo parecidb al hecho de coger un subconjunto del conjunto total de posibilidades para el estado de cosas actual. Esto significa que la esencia de la proposición es excluir al momento de afimar, pues sostener algo, es sostener que aquellas posibilidades que no se sostienen no son actuales en el mundo. El contenido de lo que uno sostiene se entiende en relación con las posibilidades que uno ha excluido, a las cuales puede considerarse como dojetos abstractos, y en tanto altemativas se tienen en arenta para definir la proposición afirmada (Stalnaker 1999: 130-149) .

Por otro lado, hay quienes sostienen que las proposiciones son entidades de carácter complejo, cuya estructura reúne de cierta manera a sus partes constituyentes ${ }^{14}$. Para ellos, el problema en tomo a la proposición consiste en especificar cuáles son estas partes, así como en poner a la luz la naturaleza del vínculo que subsiste entre ellas. En este sentido, una primera respuesta identifica las partes constituyentes de una proposición $P$ con los valores semánticos $V$ de las expresiones lingüísticas Eque configuran la oración O que expresa a la proposición $P$. De esta manera, la estnuctura de la proposición $P$ sería idéntica a la de la oración $O$. Por consiguiente, admitido que el valor semántico de un nombre es el individuo a quien hace referencia, y que el de un verbo transitivo es la relación que expresa, entonces, una oración como

(3) Juan repara su automóvil,

expresa una proposición de la estructura siguiente:

(3') $[j \mathbb{R}[a]] ;$

donde j es el individuo Juan, es decir, el valor semántico de 'Juan,' R la relación entre quien repara y lo que es reparado, es decir, el valor semántico de 'repara' y a, el automóvil de Juan, es decir, el valor semántico de 'su automóvil.' Es, sin embargo, obvio que 'su' es una 
palabra que estructura la oración dada como ejemplo y que, a pesar de esto, no se le ha tomado en cuenta como parte constituyente de la estructura de la proposición expresada por dicha oración, sino como constituyente de una expresión lingüŕstica compleja como 'su automóvil', la cual contiene dos palabras y que, por tanto, las partes de esta proposición no son idénticas a las de la oración que la expresa. Los filósofos de las proposiciones estructuradas admiten este reparo observando que muchas partes constituyentes de una proposición son valores semánticos de las palabras o frases que ocurren en la oración que la expresa, pero que hay casos excepcionales en los que ciertas palabras que configuran una oración no tienen valor semántico, de modo que su contribución a la proposición expresada resulta nula y que, además de esto, también es posible la existencia de proposiciones cuyas partes constituyentes no sean reflejo de ningún constituyente sintáctico de la oración que la expresa.

De lo anterior, se infiere que la teoría de las proposiciones estructuradas está en condiciones de justificar el hecho de que las proposiciones equivalentes pueden ser distintas, algo que la semántica de los mundos posibles se ve compelida a negar. Así, por ejemplo,

(4) Ningún círculo es cuadrado

Y

(5) Todos los solteros no están casados,

son oraciones que expresan proposiciones necesariamente verdaderas Y, por tanto, equivalentes entre sí, pero también distintas. Intuitivamente, estas proposiciones no son idénticas, no obstante que sus valores de verdad lo sean. Sus estructuras, al depender de los valores semánticos de las partes constitutivas de las oraciones que las expresan, son distintas, ya que estas oraciones lo son. Y la cuestión de cómo se unen las partes que constituyen la estructura de una proposición se sigue de cómo se unen las palabras de la oración que la exprese, así como de la relación entre estas palabras y sus correspondientes valores semánticos ${ }^{15}$.

Ia postura algebraica, a diferencia de la de los mundos posibles y de las proposiciones estructuradas, no reduce las proposiciones a entidades extensionales tales como funciones o $n$-tuplos. Sostiene que 
las proposiciones son por naturaleza conjunciones, negaciones, predicaciones, generalizaciones existenciales, etc. Esta respuesta es lo más intuitiva a la pregunta por la esencia de la proposición y, como se aprecia, su carácter no es reduccionista. Entre los argumentos a su favor, figura el de su mayor plausibilidad frente a sus competidoras, y otro que consiste en tomar una perspectiva intensional frente a la lógica extensional, considerando a propiedades, relaciones y proposiciones como entidades sui generis, y analizando el comportamiento de éstas con respecto de las operaciones lógicas fundamentales, es decir, la conjunción, la negación, la predicación singular, la generalización existencial, etc. Este análisis se lleva a cabo mediante el estudio de estructuras intensionales modelos, las mismas que constan de un dominio, un conjunto de operaciones lógicas y un conjunto de funciones de extensionalización posible. El dominio se divide en subdominios de particulares, proposiciones, propiedades, relaciones binarias, relaciones temarias, etc., todos tomados como entidades primitivas. El conjunto de operaciones lógicas incluye las fundamentales así como las auxiliares. Las funciones de extensionalización posible asignan una extensión a cada uno de los ítemes del dominio. Por ejemplo, si H es la función de extensionalización, y conj la qperación lógica fundamental de conjunción, entonces para todas las proposiciones p y q en el dominio en cuestión, $H(c o n j(p, q))$ es verdadero si y sólo si $H(p)$ es verdadero y $\mathrm{H}(\mathrm{q})$ es verdadero. De manera similar se procede en el caso del resto de operaciones lógicas fundamentales y auxiliares ${ }^{16}$.

Los párrafos anteriores ofrecen una vista panorámica de la variedad de respuestas que pueden aducirse a la pregunta por la esencia de la proposición. Como suele suceder, ninguna de ellas es inmune a ser objetada. Se ha visto, por ejemplo, que al identificar a las proposiciones como funciones siguiendo a la semántica de los mundos posibles, uno está compelido a afirmar que todas las proposiciones equivalentes son idénticas. Quien, por su lado, se adhiera a la teoría de las proposiciones estructuradas afirmará que la proposición refleja de una u otra manera la estructura gramatical de la oración que la expresa en determinado contexto y de este modo afinmará también que las partes de la oración son partes de la proposición, y no meros medios por los cuales ésta se expresa; pero es claro que una misma proposición puede expresarse 
de diferentes maneras y de esto, se seguiría que una misma proposición tiene diversas estructuras. Para terminar, la explicación algebraica concede demasiada importancia a su mayor plausibilidad frente al resto, y se exime de explayarse en detalles técnicos. Pero no es mi propósito argumentar a favor de estas objeciones, sólo fue necesario haber expuesto la disimilitud de respuestas posibles a la pregunta por la esencia de la proposición, y que todas aceptan que las proposiciones son los entes privilegiados para la atribución de propiedades tales como verdad, falsedad, necesidad, posibilidad e imposibilidad. Mi interés estuvo circunscrito a este punto; no importa, pues, mi compromiso ontológico con respecto a las proposiciones.

\section{Notas}

1. El texto que presento bajo este título ha sido adaptado del correspondiente a una sección introductoria de mi actual investigación "El concepto de verdad en las ciencias naturales" . En este proyecto, auspiciado por el Consejo Superior de Investigación de la Universidad Nacional Mayor de San Marcos, me acompañan los profesores Gilberto Bustamante Guerrero y Marino Llanos Villajuán, y los estudiantes José Antonio Tejada Sandoval y Joseph David de Jesús Villena Saldaña. Considero necesario expresar un reconocimiento especial a este último por su estrecha colaboración y gentiles sugerencias.

2 Sin embargo, debe considerarse que 'amarillo' puede ir en la posición gramatical de un predicado, mientras que 'ser amarillo' $\multimap$ la 'amarillez' -, no. Este fenómeno origina una distinción entre concepto y propiedad; según ésta, el concepto es una propiedad que puede ser empleada como predicado, mientras que la propiedad en sentido estricto no puede ser empleada como predicado, al menos no en una oración con sujeto y verbo idénticos al del caso anterior (David Wiggins 1984) concluye que los conceptos no pueden identificarse con las propiedades, aunque sí con ciertas formas o rasgos universales. De acuerdo con su argumento, un concepto puede combinarse con una cópula y con un nombre para producir una oración significativa. La propiedad, por su lado, de combinarse con los mismos elementos no produce una oración equivalente. Por ejemplo, las siguientes oraciones no son equivalentes: 'Jesús es humano,' en donde 'humano' es el concepto, y 'Jesús es humanidad,' en donde 'humanidad' es la propiedad. Strawson (2000) ofrece un ejemplo que dejaría sin validez la argumentación de Wiggins. Si como éste sugiere, el concepto y la propiedad no hacen referencia al mismo objeto, entonces 'Sócrates es sabio' y 'Sócrates tiene sabiduría,' aparte de no ser equivalentes, serían oraciones que expresan cosas disímiles. Esto, por supuesto, aparece como intuitivamente falso, ya que 'ser sabio' y 'tener sabiduría' tienen, de alguna u otra manera, idéntico valor semántico. Para Strawson, 'concepto' y 'propiedad' son expresiones alternativas, de modo que cuando una de ellas es copulada predicativamente, entonces, si es especificada mediante un adjetivo, es normalmente copulada por el uso del verbo 'ser' , y si es especificada por un nombre abstracto, es normalmente copulada por el verbo 'tener' o por algún verbo afín (Salmon 1989). 
3 Un argumento a favor del predicado como término singular es que los predicados pueden ser nominalizados de modo que ocupen la posición de sujeto en una oración, por ejemplo, amarillez, sabiduría, humanidad. En estas circunstancias, no designan un conjunto de cosas y, por tanto, es natural asumir la uniformidad semántica y tomar el predicado correspondiente como si designara propiedades cuando ocurren en la posición de predicado (McGrinn 2000: 52-68) .

4 La siguiente clasificación ha tomado como pauta la efectuada por Chris Swoyer (2000). Advierto, sin embargo, que me he apartado de esta clasificación cuando lo he creído conveniente. Por mencionar un ejemplo, ella da dieciocho clases de propiedades, mientras que yo doy doce.

5 Para fines del artículo, es indiferente que la teoría de los colores presupuesta por este ejemplo sea objeto de controversia.

6 Habrá quien replique que, al estudiar la naturaleza de determinada propiedad, antes que definir el conjunto de entes de los cuales se predica con validez, debe empezarse estableciendo de qué clase de propiedad se trata, para que, de inmediato -y ya facilitada la labor-, se proceda a ubicar las entidades deseadas. Lamentablemente, de aplicarse este método en el caso de la verdad, no se obtendría un resultado semejante, pues, aunque se conozca que es una propiedad relacional -como ciertamente lo es-, los elementos de juicio para inferir cuál sea el conjunto de entidades que le concieman no serían suficientes. Entonces, sería necesario continuar elaborando distinciones, esta vez, indicándose qué tipo de relación es la verdad y proponiéndose, por tanto, una teoría de la verdad recién empezada la investigación, una que, dadas las circunstancias, podría ser bien la de correspondencia, bien la coherentista. Como se aprecia, este artículo evade el desorden metodológico en su discurir.

7 Esto, por supuesto, no se aplica al caso de la infinitud. Delimitándose el conjunto de lo que puede ser infinito, se sabrá qué es lo que no puede serlo, y, por tanto, lo que es necesariamente finito. En el campo de lo que puede ser infinito habrá, sin embargo, entes que sean ora finitos ora infinitos; mientras que en lo que toca a la verdad, si algo puede ser verdadero también puede ser falso, de ahí que si algo no pueda ser verdadero tampoco pueda ser falso.

8 Esta afirmación aparenta estar implicada por la teoría de la verdad por correspondencia, pero si se repara en lo que sostiene la teoría coherentista, se caerá en cuenta de que no es así. Según ésta, la proposición o proposiciones que determina (n) la verdad de tal o cual proposición no guarda (n) necesariamente ninguna similitud formal con esta última. De esta manera, afirmar que la verdad de los entes que son verdaderos se establece en virtud de entes de estructura formal disímil, puede presuponer ya sea a la teoría de la verdad por correspondencia o a la coherentista. No violo, por tanto, la pauta metodológica consignada en la nota 9.

9 Por cuestiones de argumentación, no consideraré la eventual crítica de un deflacionista, quien niega que la verdad sea una propiedad, algo que constituye la primera asunción de este artíarlo.

10 Quizá se señale una contradicción, ya que antes sostuve que los entes físicos no son verdaderos, y ahora califico a las oraciones de verdaderas, aun cuando las haya definido como entes físicos. En realidad, el hecho de que un ente sea físico no es condición suficiente para privársele de la pasibilidad de ser verdadero o falso. Resulta, sin embargo, que la oración es el único ente físico que conlleva una proposición y todas las características formales de ésta, y esto es lo que da valor de verdad a un ente cualquiera. De esta manera, puede afirmarse que todo ente físico que no sea una oración no es ni verdadero ni falso. 
Coservar entes físicos nos revela cuestiones acerca de ellos, como que sea rojo, etc. Pero, al ver una manzana, yo no leo 'esta manzana es roja;' es decir, la manzana no es una oración, ni tiene como características intrínsecas proposiciones, como tampoco lo es una mano ni las hojas de té.

11 Supóngase que el sujeto $H$, tendido sobre un campo, mira al cielo. Este sujeto sostiene que allí doserva lo siguiente:

(h) Lima es la capital de Perú.

La configuración providencial de un conjunto de nubes dio como resultado una oración en lengua castellana. De acuerdo con cierto documento, esta oración es verdadera. El sujeto H no sostendrá que la expresión (h) (usted debe hacer de cuenta que los caracteres de (h) son nubes), sea verdadera y tampoco falsa, pues esto implicaría que, aparte de las palabras, lo sean también las nubes.

12 A propósito de esto, Soames (1999: 16) un deflacionista moderado, sugiere que los significados pueden ser funciones de contextos de expresión de proposiciones ya expresadas. Para evitar la confusión entre proposición y significado, emplea los términos 'oración ocasional' y 'oración etema' . El significado de una oración ocasional es una función que asigna diferentes proposiciones como valores a los diferentes contextos como argumentos. El significado de una oración etema es una función que asigna la misma proposición a todos los contextos. En el último caso, la función es constante, y podría dar pie a dbviar los contextos e identificar el significado de una oración etema con la proposición que ella expresará siempre, lo que no se aprecia en el caso de las oraciones ocasionales.

13 Dejo de lado la postura materialista con respecto a las proposiciones, es decir, el concretismo. Según el concretismo, las proposiciones son objetos concretos, específicamente inscripciones cerebrales. Resulta claro que una línea de pensamiento como ésta se encuentre expuesta a graves objeciones, tales como que de no haber existido seres humanos $y$, por tanto, ningún cerebro, tampoco habrían existido proposiciones, y entonces, a pesar de que un estado de cosas semejante haga verdadera la proposición de que no existen seres humanos, tampoco podría afirmarse de que no existen seres humanos. Además, el concretista es por naturaleza determinista y, así, cree que hay proposiciones necesariamente verdaderas, sin embargo, su ontología lo forzará a negar esto último, pues identifica a las proposiciones con objetos concretos, y, en consecuencia, contingentes; por ello, estaría forzado a afimar que no hay proposiciones necesariamente verdaderas, y que tampoco lo sea la proposición 'las proposiciones son inscripciones cerebrales' . La inconsistencia se puede probar mediante varias abjeciones más. Al respecto de las aducidas y otras, cf. Alvin Plantinga (2003: 229233)

14 Los representantes más conspicuos de esta postura son Soames (1987) y Salmon (1989) quien habla de 'proposiciones russellianas estructuradas' .

15 Jeffrey C. King (1996) ha desarrollado recientemente una teoría sobre este tema, la cual, a juicio de su autor, no permitiría que se dude de la existencia entitativa de las proposiciones.

16 En este párrafo he seguido a George Bealer (1998), artículo seminal con respecto a la explicación algebraica para la teoría de la proposición.

\section{Referencias bibliográficas}

ARISTÓIEIES (1967) : "De Interpretatione". Obras. Aguilar. Madrid ; pp. 255-271. BEALER, George (1998) : "Propositions". Mind, 107; pp. 1-32. 
CARIWRIGH, Richard (1962) : "Propositions". En R. J. Butler (editor) Analytical Philosqphy. Basic Bladkwell. Oxford; pp. 81-103.

GOODNAN, N. Y W. V. QUINE (1947) : "Steps toward a constructive nominalism." Jamal of Symbolic Logic, 12; p.p. 97-122.

KANI, Immanuel (1998) : Kritik der reinen Vemunft. Akademie Verlag. Berlín.

KING, Jeffrey (1996) : "Structured Propositions and Sentence Structure". Joumal of Philosophical Logic, 25; pp. 495-521.

LOCKE, John (1987) : An Essay Conceming Himan Understanding. Clarendon Press. Oxford.

IFWLS, David (1973) : Canterfactuals. Harvard University Press, Cambridge.

MbGTNN, Colin (2000) : Logical Properties. Oxford University Press. New York.

PLANIINGA, Alvin (2003) : Essays in the Metaphysics of Mbolality. Oxford University Press. New York.

QIINE, W. V. (1969) : "Natural Kinds". EnN. Rescher et alii (editors) Essays in Honor of Carl G. Hempel. D. Reidel, Dordredht; pp. 5-23.

QUINE, W,V. (1960) : hord and Object. MIT Press. Cambridge.

SAIMaN, Nathan (1989) : "Tense and Singular Propositions" . En J. Almog, J. Perry Y H. Wettstein (editors) Themes Fram Kaplan. Oxford University Press. New York; pp. 331-392.

SOAMES, Scott (1987) : "Direct Reference, Propositional Attitudes and Semantic content". Philosquhical Topics, 15; pp. 47-87.

SOAMES, Scott (1999) : Understanding Thuth. Oxford University Press. New York.

SIAINAKER, Robert (1999) : Context and Content, Oxford University Press. New York. SIAINAKER, Rdbert "Propositions." A. MacKayy D. Merrill (editors), Issues in the Philosqphy of Language. Yale University Press. NewHaven; pp. 79-91.

SIRAWSON, P. F. (2000) : Entity and Identity. Oxford University Press. New York.

SWOYER, Chris (2000): "Properties". Edward N. Zalta (editor). The Stanford Encyclqpedia of Philosqphy. «http://plato.stanford.edu/archives/win 2000/ entries/ pro-perties/>.

WIGGMNS, David (1984) : "The Sense and Reference of Predicates: A Rumning Repair to Frege's Doctrine and a Plea for the Copula". Philosqphical Quarterly, 34; pp. 311328. 